\title{
Atomic-scale Insights of Cation Diffusion into Multivalent Battery Cathodes
}

\author{
Prakash Parajuli ${ }^{1}$, Bob Jin Kwon², Sanghyeon Kim², Baris Key ${ }^{2}$, John Vaughey ${ }^{2}$ and Robert Klie ${ }^{1}$
}

${ }^{1}$ University of Illinois at Chicago, Chicago, Illinois, United States, ${ }^{2}$ Argonne National Laboratory, Lement, Illinois, United States

In early 2021, General Motors announced that it will end the production of its cars, trucks, and SUVs with diesel- and gasoline-powered engines by 2035. This and other similar trends of transition necessitates the widespread deployment of high energy charge storage system and the integration of more renewable energy into the grid. Currently, Li-ion batteries have been serving the majority of the demand of rechargeable energy sources. However, the long-term concerns of high cost, limited reserves of lithium/cobalt, and safety of Li-ion batteries have driven the researchers to search for alternative energy storage solutions. Among them, multivalent cations such as $\mathrm{Mg}^{2+}, \mathrm{Ca}^{2+}, \mathrm{Zn}^{2+}, \mathrm{Ni}^{2+}, \mathrm{Al}^{3+}$ are considered as potential candidates for beyond $\mathrm{Li}$-ion batteries considering not only their ability to transfer more than one electron thereby facilitating higher energy density but also the possibility of using corresponding metals as the anode of the battery system for their energy density and improved safety. ${ }^{1-3}$

Despite tremendous efforts in the field, the progress towards the development of multivalent battery systems is often hindered by a lack of suitable cathode chemistries that show good reversibility of ion intercalation. The main challenge to overcome is sluggish solid-state diffusion for multivalent ions and the desolvation of solvent ligands at the cathode surface. ${ }^{1}$ It appears that the sluggish intercalation chemistry during electrochemical cycling is mostly due to structural changes and phase transitions hindering the mobility and reversibility of the cations. ${ }^{4,5}$ Hence, the systematic and thorough understanding of those intercalated structures at the atomic-scale is necessary if multivalent ion-based batteries are to be turned from laboratory cells into a commercial product.

In this contribution, we will provide insights into the atomic-level structure and spectroscopy of the multivalent cathodes after discharge, which are mostly undetectable during bulk characterization. In particular, structural changes in $\mathrm{MgCrMnO}_{4}$ after $\mathrm{Mg}$ discharge and $\mathrm{FePO}_{4}$ after $\mathrm{Ca}$ discharge are explored by employing atomic-resolution imaging and electron energy loss spectroscopy. All the electronmicroscopy studies were performed in an aberration-corrected JEOL JEM-ARM200CF, equipped with a cold field emission gun, operated at $200 \mathrm{kV}$. Furthermore, battery cathode samples were prepared inside an Ar-filled glovebox to avoid any chance of sample degradation by air exposure. Results show that $\mathrm{Mg}$ intercalation into $\mathrm{MgCrMnO} 4$ spinels results in the formation of a rocksalt surface layer, while $\mathrm{Ca}$ intercalation into polyanionic compounds proceeds via a core-shell process. These results guide battery scientists to better select and design the components of the battery to optimize the performance of the system.

Figure 1 shows atomic resolution images of pristine and $\mathrm{Mg}$ discharged $\mathrm{MgCrMnO}_{4}(\mathrm{Mg}$ discharged after the extraction of $\mathrm{Mg}$ ). Close analysis of the discharged particle shows two distinct crystallographic phases; spinel in region I, having a diamond-shaped unit cell, whereas a defective rocksalt on region II showing empty tetrahedral sites (8a; Wyckoff notation) and occupied octahedral sites (16c; Wyckoff notation), which are empty in the region I. Furthermore, the contrast of the atomic columns across various columns is almost homogenous in region II indicating a defective rocksalt phase on the particle's surface upon $\mathrm{Mg}$ discharge. 
Atomic-resolution images and EELS spectroscopy of the Ca discharged $\mathrm{FePO}_{4}$ are summarized in Figure 2. As illustrated in Figs 2(a,b), results show that Ca mostly intercalates on the surface of the particle showing 1) diminishing trend of $\mathrm{Ca} \mathrm{L}$ edge signals and 2) gradual decrease of $\mathrm{Fe} \mathrm{2+}$ and increase of $\mathrm{Fe} 3+$ from the surface to the bulk of the particle. Furthermore, the annular bright-field image of the $\mathrm{Ca}$ discharged $\mathrm{FePO}_{4}$ clearly shows the decrease in the atomic contrast of the expected $\mathrm{Ca}$ atomic columns from the surface to the bulk of the particle. These results demonstrate the core-shell pattern of $\mathrm{Ca}$ intercalation into $\mathrm{FePO}_{4}{ }^{6}$

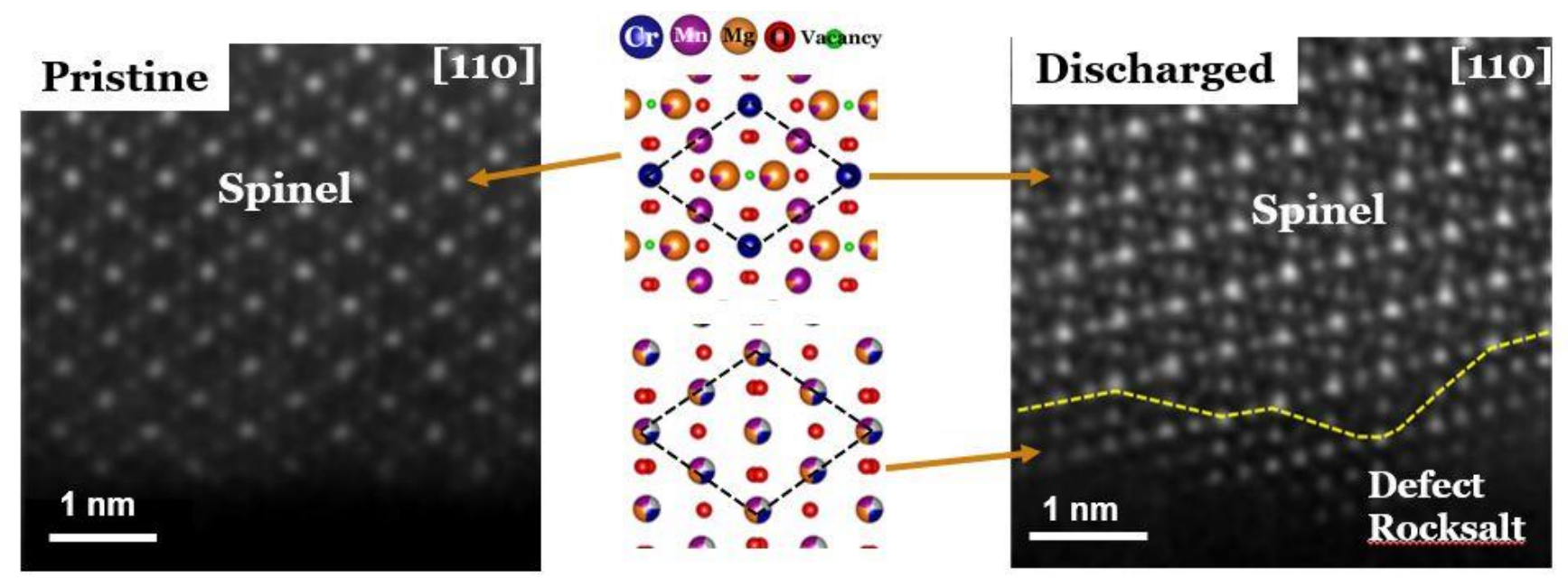

Figure 1. Figure 1: STEM-HAADF images of pristine and $\mathrm{Mg}$ discharged $\mathrm{MgCrMnO} 4$ showing spinel phase in pristine materials, whereas defect rocksalt phase on the surface of the discharged structure. 


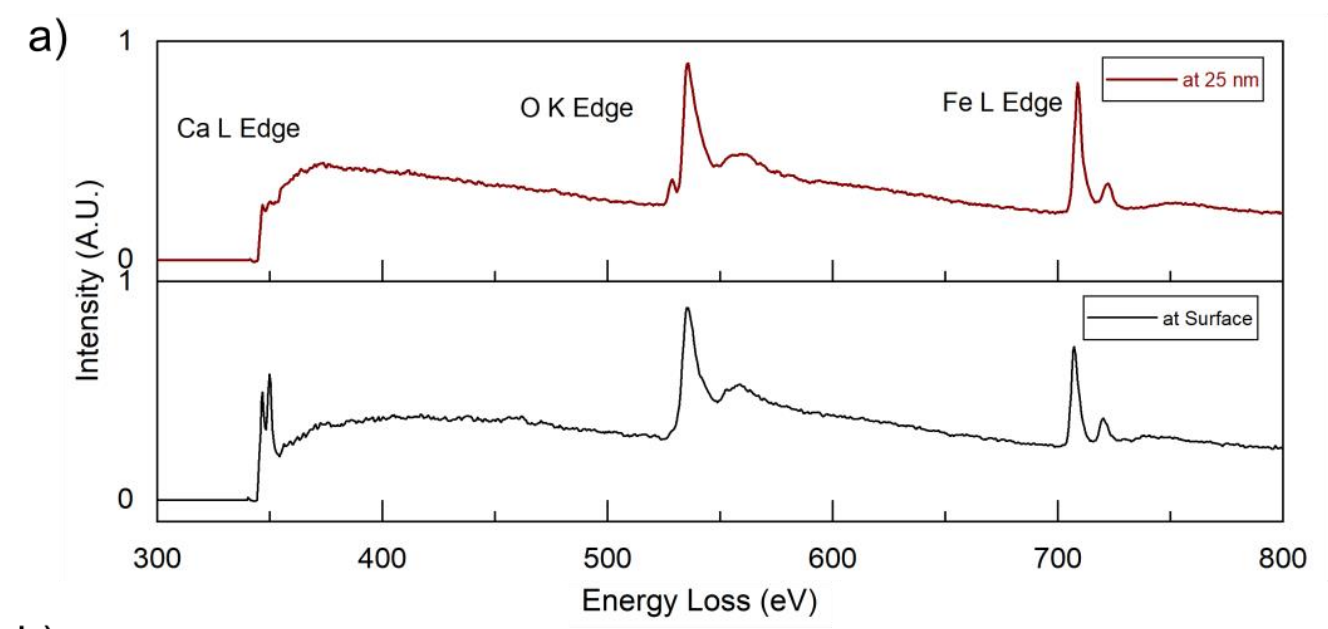

b)

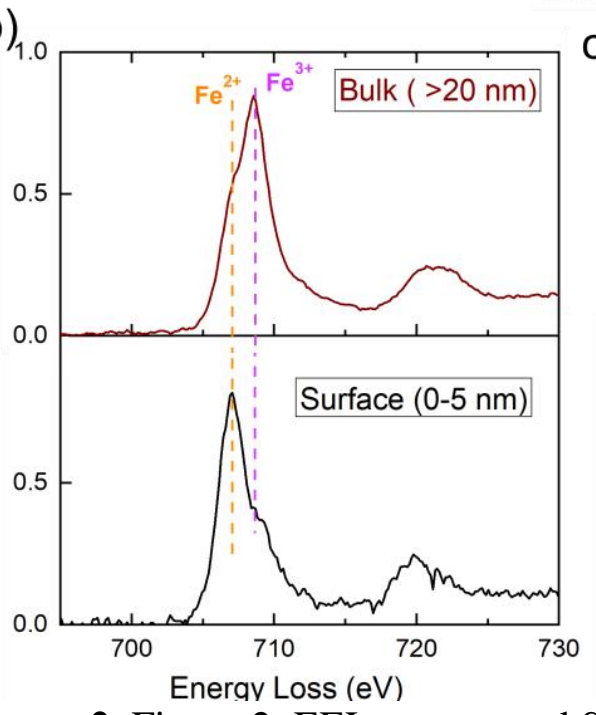

c) [110]

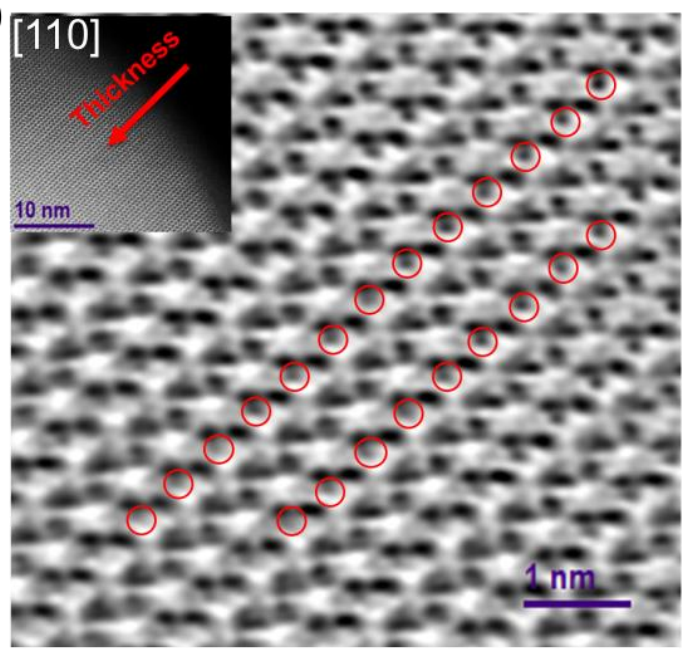

Figure 2. Figure 2: EEL spectra and STEM-ABF image of Ca discharged FePO4 showing the core-shell pattern of $\mathrm{Ca}$ intercalation. (a) EEL spectra from surface and bulk showing prominent $\mathrm{Ca}$ signals from the surface, signals diminished with an increase in thickness. b) Magnified view of the Fe L-edge showing mostly $\mathrm{Fe} 2+$ on the surface and $\mathrm{Fe} 3+$ on the bulk. c) STEM-ABF image showing the decrease in the atomic contrast of Ca columns with an increase in thickness.

\section{References}

1. Canepa, P. et al. Odyssey of Multivalent Cathode Materials: Open Questions and Future Challenges. Chem. Rev. (2017).

2. Kwon, B. J. et al. High Voltage Mg-Ion Battery Cathode via a Solid Solution Cr-Mn Spinel Oxide. Chem. Mater. 32, (2020).

3. Kim, S. et al. High-Voltage Phosphate Cathodes for Rechargeable Ca-Ion Batteries. ACS Energy Lett. 5, (2020).

4. Okamoto, S. et al. Intercalation and Push-Out Process with Spinel-to-Rocksalt Transition on Mg Insertion into Spinel Oxides in Magnesium Batteries. Adv. Sci. 2, 1500072 (2015).

5. Parajuli, P. et al. Direct Observation of Electron Beam-Induced Phase Transition in MgCrMnO4. Chem. Mater. 32, 10456-10462 (2020). 
6. This work was supported by the Joint Center for Energy Storage Research (JCESR), an Energy Innovation Hub funded by the U.S. Department of Energy, Basic Energy Sciences. 